\title{
CHARACTERIZATIONS OF BOUNDEDNESS FOR GENERALIZED FRACTIONAL INTEGRALS ON MARTINGALE MORREY SPACES
}

\author{
EIICHI NAKAI AND GAKU SADASUE
}

Abstract. On generalized martingale Morrey spaces we give necessary and sufficient conditions for the boundedness of generalized fractional integrals as martingale transforms.

Mathematics subject classification (2010): 60G46, 60G42, 46E30, 42B35.

Keywords and phrases: Martingale, Morrey space, fractional integral.

\section{REFERENCES}

[1] D. R. Adams, A note on Riesz potentials, Duke Math. J., 42, 4 (1975), 765-778.

[2] D. L. Bur Kholder, Martingale transforms, Ann. Math. Stat., 37 (1966), 1494-1504.

[3] J.-A. Chao And H. Ombe, Commutators on dyadic martingales, Proc. Japan Acad., 61, Ser. A (1985), 35-38.

[4] ERIDANi, H. GunawAn AND E. NAKAI, On generalized fractional integral operators, Sci. Math. Jpn., 60 (2004), 539-550.

[5] ERIDANi, H. GunAwan, E. NAKAI AND Y. SAWANo, Characterizations for the generalized fractional integral operators on Morrey spaces, Math. Inequal. Appl., 17, 2 (2014), 761-777.

[6] H. Gunawan, A note on the generalized fractional integral operators, J. Indones. Math. Soc., 9, 1 (2003), 39-43.

[7] L. I. Hedberg, On certain convolution inequalities, Proc. Amer. Math. Soc., 36 (1972), 505-510.

[8] Z. HAO AND Y. JiAO, Fractional integral on martingale Hardy spaces with variable exponents, Fract. Calc. Appl. Anal. 18, 5 (2015), 1128-1145.

[9] E. NAKAI, On generalized fractional integrals, Taiwanese J. Math., 5, 3 (2001), 587-602.

[10] E. NAKAI, On generalized fractional integrals on the weak Orlicz spaces, $\mathrm{BMO}_{\phi}$, the Morrey spaces and the Campanato spaces, Function spaces, interpolation theory and related topics, 389-401, de Gruyter, Berlin, 2002.

[11] E. NAKAI, A generalization of Hardy spaces $H^{p}$ by using atoms, Acta Math. Sinica 24 (2008), $1243-$ 1268.

[12] E. NAKai And G. Sadasue, Martingale Morrey-Campanato spaces and fractional integrals, J. Funct. Spaces Appl., 2012 (2012), Article ID 673929, 29 pages.

[13] E. Nakai, G. Sadasue and Y. Sawano, Martingale Morrey-Hardy and Campanato-Hardy Spaces, J. Funct. Spaces Appl., 2013 (2013), Article ID 690258, 14 pages. DOI:10.1155/2013/690258

[14] G. Sadasue, Fractional integrals on martingale Hardy spaces for $0<p \leqslant 1$, Mem. Osaka Kyoiku Univ. Ser. III Nat. Sci. Appl. Sci., 60, 1 (2011), 1-7.

[15] H. TANAKA AND Y. TERASAWA, Positive operators and maximal operators in a filtered measure space, J. Funct. Anal. 264, 4 (2013), 920-946.

[16] C. WATARI, Multipliers for Walsh Fourier series, Tohoku Math. J. 16 (1964), 239-251.

[17] F. WeISZ, Martingale Hardy spaces and their applications in Fourier analysis, Lecture Notes in Mathematics, 1568, Springer-Verlag, Berlin, 1994. 